\title{
Le bouchon vaseux estuarien : filtre actif ou passif
}

\author{
The estuarian silt plug : active or passive filter
}

\author{
par Louis A. Romaña
}

IFREMER, Département Polluants Chimiques*

* Zone Industrielle de Brégaillon, B9 330, 83507 La Seyne sur Mer.

Direction de l'Environnement Littoral et de l'Aménagement.

The estuarial environment shows certain specific features which must absolutely be taken into account as part of their management. The tide is specially responsible of a large amount of fine sedimentary material named in France as "bouchon vaseux (split plug)". We shall explain in this paper, the origin, the importance and the environmental consequences of this turbid mass, and try to find out some simple ideas about the operational management of the estuarial sediments.

\section{INTRODUCTION}

Parmi les grands apports continentaux, les fleuves jouent un rôle primordial. Pendant leur important parcourt, ils accumulent et transportent sous forme dissoute et particulaire, inorganique ou biologique, des concentrations souvent croissantes d'éléments susceptibles d'enrichir, voire de modifier les cycles biogéochimiques côtiers.

A leur arrivée en mer, et selon le niveau énergétique de celle-ci, ces apports massiques peuvent subir de profondes modifications, tant qualitatives que quantitatives. Dans cette confrontation entre le fleuve et la mer naîtra un milieu spécifique, doté de caractéristiques propres qui en font des sites exceptionnellement riches en termes de production biologique. Par contre, étant aussi le point d'aboutissement et d'accumulation des déchets et rejets de la plupart des activités anthropiques qui ont lieu sur le bassin versant, l'accumulation de ces apports risque d'entraîner de sérieux problèmes pour la qualité du milieu :

C'est l'intensité des courants de marée qui différencie les estuaires des deltas. Dans le cas de faibles marées, c'est l'énergie du fleuve qui domine celle de la mer, et le système se construit dans le domaine fluvial. Dans le cas contraire, surtout dans les zones à forts marnages, c'est la mer qui freine l'énergie du fleuve, l'obligeant à composer. C'est ainsi que naissent les estuaires macrotidaux.

Les deltas, du fait de leur progression géographique ont fortement limité l'installation permanente des hommes. De ce fait ils sont beaucoup moins touchés par les aménagements.

Par contre les estuaires, où l'installation de l'homme dans un milieu physique plus stable était un peu plus facile, ont dû subir à des degrés divers de profondes modifications géomorphologiques. Nous pouvons citer en France la construction de ports, de digues, de ponts, de centrales nucléaires, l'extraction de sables et graviers, les dragages d'entretien...
Tout ceci vient s'ajouter ou interférer avec les problèmes de la qualité des eaux, du fait des apports du fleuve ou des rejets locaux.

Comme principales conséquences on peut citer la restriction au passage des poissons migrateurs (barrages, oxygène dissous), le problème de la gestion des rejets urbains et industriels, la gestion des prises d'eau potable, la dégradation des peuplements naturels, la contamination de la ressource... Beaucoup d'autres exemples peuvent être cités.

\section{II $\square$ UN ESTUAIRE MACROTIDAL}

Le terme estuaire vient du latin aestus, qui signifie marée. Sur le plan morphologique, l'estuaire est défini comme une vallée fluviale ouverte sur l'océan. Sur le plan écologique, l'estuaire est un écosystème qui constitue une interface d'échanges entre les domaines continentaux et marins.

Ces milieux sont complexes et les auteurs ne s'accordent pas sur une définition unique de l'estuaire : selon les disciplines, l'estuaire ne correspond pas à la même entité spatiale. Ainsi, en 1967 pour Pritchard (physicien), l'estuaire est la zone dans laquelle les eaux d'origine marine sont diluées de manière mesurable par les eaux douces. Cette définition sert de base aux études estuariennes qui ont été principalement focalisées sur les secteurs soumis à l'influence saline.

Dès que l'on engage des études écologiques en milieu estuarien, et que l'on requiert l'intervention de scientifiques de diverses disciplines, il apparaît clairement dans la définition de l'estuaire le besoin d'intégrer la zone soumise aux seuls effets de la marée. C'est Fairbridge en 1980 qui propose une définition et un découpage de l'estuaire incluant la zone non salée à marée dynamique : «Un estuaire est un bras de mer pénétrant une vallée fluviale jusqu'à la limite amont de propagation de la marée ». Il est habituellement divisible en 3 secteurs :

_ Un bas estuaire ou estuaire marin : 
- Un estuaire moyen, sujet à d'importants mélanges entre eaux douces et eaux salées ;

- Un haut estuaire ou estuaire fluvial, caractérisé par de l'eau douce mais sujet aux actions quotidiennes de la marée.

Les limites entre ces trois secteurs sont variables et sujettes aux constantes variations du débit fluvial.

L'étendue de ces différents secteurs dépend, à la fois, du cadre géomorphologique et du rapport entre le débit du fleuve et l'importance des marées.

Ces deux phénomènes, liés entre eux, caractérisent l'estuaire macrotidal. Ils lui conferent une place à part dans les différentes zones géographiques de notre littoral et révèlent l'action de plusieurs processus complexes, aux conséquences importantes sur la qualité des eaux estuariennes.

A certaines périodes les eaux estuariennes deviennent extrêmement turbides, au point que si l'on y plonge sa main elle n'est plus visible au-delà de quelques centimètres. Ce ne sont pas les fleuves qui sont directement responsables de cette turbidité, puisque si l'on remonte vers l'amont de quelques dizaines de kilomètres on retrouvera facilement des eaux plus claires.

On trouve aussi souvent dans les régions proches des zones turbides de ces estuaires, des vasières subtidales et intertidales bien développées, biologiquement riches, où s'installent à basse mer des oiseaux pour s'alimenter et, à pleine mer, certains juvéniles de poissons.

En fait, c'est la présence d'un gradient de salinité et la capacité du milieu à stocker les vases, deux conséquences de l'action de la marée, qui identifient un estuaire macrotidal. L'un est la conséquence des processus hydrodynamiques, l'autre résulte de l'action de mécanismes hydrosédimentaires.

\section{II.1 Les processus hydrodynamiques}

Dans nos régions du Nord de l'Europe, la marée est de type semi-diurne : Son amplitude varie au cours du mois avec une périodicité de 14,5 jours correspondant à une demi-lunaison. En vives eaux l'amplitude maximale à l'embouchure des estuaires de la Manche varie entre 5 et 10 mètres.

Les ondes de marée océaniques présentent une symétrie flot/jusant et peuvent être, dans de nombreux cas, assimilées à des sinusoïdes. Aux abords des côtes et à mesure que les fonds sont moins profonds ces ondes de marée subissent de nombreuses déformations.

C'est l'énergie introduite par la marée qui façonne I'intérieur de l'estuaire et lui donne sa forme "évasée». A la force des eaux du fleuve s'oppose ou se conjugue, au cours de la marée, la puissance du flot ou du jusant.

La conjonction du débit et de la marée induit dans un estuaire macrotidal, une circulation résiduelle des eaux en deux couches. Ceci conduit à la notion de point nodal où disparaissent les courants résiduels vers l'amont. En général, ce point nodal marque la pénétration maximale de la salinité.

La dispersion introduite par le mouvement périodique des eaux vers l'amont et vers l'aval fait que l'eau de mer est diluée d'une façon mesurable avec de l'eau douce. Nous retrouvons ici la notion de mélange et de gradient de salinité. De même, ce processus de mélange a pour conséquence la remontée possible d'une partie des substances dissoutes rejetées dans l'estuaire.

\section{II.2 Les processus hydrosédimentaires}

C'est l'interaction des courants de marée de surface et des frottements induits par les courants de marée sur le fond qui engendre un piégeage naturel de sédiment en suspension (ou matières en suspension $=$ MES). Le stock sédimentaire ainsi piégé est désigné par le terme de "bouchon vaseux " ou «maximum de turbidité" et explique les fortes turbidités rencontrées en milieu estuarien. Les concentrations en MES au sein des bouchons vaseux sont de 100 à 500 fois plus importantes que celles que l'on rencontre dans les cours d'eau ou en mer ouverte.

En Seine, la masse sédimentaire piégée au niveau du bouchon vaseux a été évaluée entre 20000 et 400000 tonnes, en Loire elle est estimée autour de 800000 tonnes, en Gironde, de l'ordre de 5 millions de tonnes.

Le bouchon vaseux se déplace d'amont en aval suivant le débit du fleuve et le moment de la marée (flot ou jusant).

Quant au stock en suspension, il augmente et diminue en fonction du cycle lunaire de la marée. La partie déposée de ce stock porte le nom de crème de vase : elle est maximale en période de mortes eaux. Ce stock, dont la concentration en sédiments est très forte (jusqu'à $100 \mathrm{~g} / \mathrm{l}$ ), joue probablement un rôle fondamental dans une éventuelle dégradation de la qualité du milieu.

\section{III — LA SITUATION DES ESTUAIRES EN FRANCE}

La France dispose sur la façade Manche-Atlantique de plus d'une centaine d'estuaires, de tailles extrêmement variables dont seulement $5 \%$ sont des grands fleuves pour lesquels les débits moyens annuels dépassent les $50 \mathrm{~m}^{3} \cdot \mathrm{s}^{-1}$, alors que pour les trois quarts d'entre eux les débits n'excèdent pas les $10 \mathrm{~m}^{3} \cdot \mathrm{s}^{-1}$.

\section{IV — PRINCIPAUX ESTUAIRES FRANÇAIS}

La France possède trois estuaires de grande taille qui sont, par ordre décroissant : La Gironde $\left(625 \mathrm{~km}^{2}\right.$ de surface), La Loire $\left(60 \mathrm{~km}^{2}\right)$ et la Seine $\left(50 \mathrm{~km}^{2}\right)$. Leur histoire leur a conféré des caractéristiques propres.

La Gironde, dont le bassin versant est relativement peu peuplé et peu industrialisé, a conservé un équilibre naturel qui se manifeste par un climax géomorphologique bien établi et une faible pollution, que sa taille importante contribue de plus à renforcer.

La Loire draine un bassin versant plus développé et plus riche, ce qui a conduit à aménager non seulement son estuaire mais aussi son environnement. Ces aménagements ont en particulier été réalisés en vue de faciliter le passage des grands navires (Ports de Nantes et de Saint-Nazaire) et d'extraire, en amont, les sables et les graviers. Ceux-ci ont changé fondamentalement la morphologie de l'amont de l'estuaire, en provoquant notamment une progression vers l'amont des eaux marines et de la turbidité. Moins de volume d'eau, plus de matière organique stockée, ceci a conduit à une désoxygénation progressive des eaux atteignant l'anoxie en période estivale.

L'estuaire de la Seine est le point d'arrivée en mer des eaux d'un bassin versant de $78650 \mathrm{~km}^{2}$ de surface, où se trouvent $20 \%$ de la population française dont l'agglomération pari- 


\begin{tabular}{|c|c|c|}
\hline $\begin{array}{l}\text { Nom de } \\
\text { l'estuaire }\end{array}$ & $\begin{array}{l}\text { Département } \\
\text { français }\end{array}$ & $\begin{array}{l}\text { Débit moyen } \\
\text { estimé }\left(\mathrm{m}^{3} / \mathrm{s}\right)\end{array}$ \\
\hline $\mathrm{Aa}$ & Pas de Calais & 6,00 \\
\hline $\mathrm{Cl}$ de Calais & & 4,75 \\
\hline Liane & & 4,85 \\
\hline Canche & & 12,40 \\
\hline Authie & Somme & 7,80 \\
\hline Baie de Somme & & 34,00 \\
\hline Bresle & & 5,20 \\
\hline Argues & Seine Maritime & 7,22 \\
\hline Scie & & 1,20 \\
\hline Seine & & 400,00 \\
\hline Risle & Eure & 20,00 \\
\hline Touques & Calvados & 13,50 \\
\hline Dives & & 15,00 \\
\hline Orne & & 27,50 \\
\hline Seulles & & 2,30 \\
\hline Aure & & 1,50 \\
\hline Vire & Manche & 21,00 \\
\hline Douve & & 8,00 \\
\hline Saïre & & 17,40 \\
\hline Divette & & 0,70 \\
\hline \multicolumn{3}{|l|}{ Ay } \\
\hline Sienne et Soulle & & 3,70 \\
\hline Sée & & 3,36 \\
\hline Sélune & & 4,30 \\
\hline Couesnon & & 5,30 \\
\hline Rance & Côtes d'Armor & 9,00 \\
\hline Arguenon & & 2,80 \\
\hline Genet & & 2,26 \\
\hline Leff et Trieux & & 7,5 \\
\hline Léguer & & 4,26 \\
\hline $\begin{array}{l}\text { Rivière de } \\
\text { Morlaix }\end{array}$ & Finistère Nord & 2,20 \\
\hline Penzé & & 2,90 \\
\hline Horn & & 0,90 \\
\hline Abers & & 2,20 \\
\hline Elorn et Aulne & & 28,60 \\
\hline Aven & & 3,80 \\
\hline Dourduff & & 0,50 \\
\hline Laîta & & 11,90 \\
\hline Gonessant & & 2,60 \\
\hline Goyen & & 1,50 \\
\hline Odet & & 8,50 \\
\hline Rivière d'Auray & & 2.12 \\
\hline $\begin{array}{l}\text { Rivière de Pont } \\
\text { L'Abbé }\end{array}$ & & 0,90 \\
\hline Scorff & Morbihan & 4,00 \\
\hline Blavet & & 21,80 \\
\hline Vilaine & & 91,00 \\
\hline Loire & Loire Atlantique & 935,00 \\
\hline Vie & Vendée & \\
\hline \multicolumn{3}{|l|}{ Auzance } \\
\hline Lay & & 2,43 \\
\hline \multicolumn{3}{|l|}{ Sèvre niortaise } \\
\hline Charente & $\begin{array}{l}\text { Charentes } \\
\text { Maritime }\end{array}$ & 50,00 \\
\hline \multicolumn{3}{|l|}{ Seudre } \\
\hline Gironde & Landes & 780,00 \\
\hline Leyre & & 5,00 \\
\hline Adour & $\begin{array}{l}\text { Pyrénées } \\
\text { Atlantiques }\end{array}$ & 250,00 \\
\hline \multicolumn{3}{|l|}{ Bidassoa } \\
\hline Nivelle & & 7,00 \\
\hline
\end{tabular}

sienne, et $40 \%$ de l'activité économique nationale. De ce fait, et depuis de nombreuses années, cet estuaire se trouve confronté à de sérieux problèmes de contamination. Par ailleurs, l'importance de son rôle de voie de communication a été à l'origine d'innombrables aménagements qui l'ont transformé en un estuaire complètement endigué et en voie de comblement. Il a radicalement perdu sa forme évasée.

\section{DES MILIEUX PASSIONNANTS}

Zone de nourricerie pour les jeunes larves de nombreuses espèces de poisson et d'oiseaux à intérêt international, régulateur des flux massiques entre le continent et l'océan, lieu de stockage de nombreux contaminants chimiques, zone de passage d'un certain nombre d'espèces de poissons migrateurs, les estuaires jouent un rôle fondamental dans les cycles chimiques et biologiques

Ces milieux spécifiques, où les gradients dans l'évolution amont aval des différents paramètres chimiques et biologiques sont exacerbés par le mélange des eaux douces et des eaux marines, et où se concentrent la plupart des problèmes liés à la dégradation du milieu côtier, est un des meilleurs laboratoires naturels pour les scientifiques.

C'est ici que l'on peut étudier le plus facilement les processus élémentaires concernant le transport sédimentaire (floculation, sédimentation, tri granulométrique, tassement...), le cycle des éléments majeurs (carbone, azote, phosphore...), les processus affectant la distribution des contaminants chimiques et bactériens, les effets biologiques... C'est ici aussi que le faible nombre d'espèces permet souvent de s'attaquer à la problématique des chaînes trophiques, à l'accumulation des contaminants, et à la qualité des ressources marines.

C'est ici que sont nés les premiers modèles mathématiques hydrodynamiques côtiers, facilités par la présence de conditions aux limites facilement maîtrisables... Progressivement on a vu se développer ceux ayant trait à la qualité des eaux. La modélisation mathématique du transport sédimentaire a émergé comme un besoin exprimé par les chimistes... On a aujourd'hui la possibilité de s'attaquer à des modèles intégrant la pluridisciplinarité, indispensable pour disposer des prémices d'une gestion intégrée des estuaires.

Les nombreuses études qui ont été conduites sur les cycles sédimentaires dans les estuaires nous ont permis de mettre en évidence un certain nombre de faits qu’il est intéressant ici de signaler.

Par exemple, les contaminants chimiques adsorbés sur les sédiments qui viennent s'accumuler dans le bouchon vaseux subissent des processus d'adsorption-désorption, de floculation, sédimentation, complexation, solubilisation, etc. De ce fait :

- Ils peuvent induire des changements dans la spéciation des contaminants. L'estuaire transforme alors la forme chimique et donc la qualité des flux contaminants vers la mer ;

- Le bouchon vaseux stocke et élimine dans les sédiments du fond de l'estuaire et/ou dans les vasières subtidales et intertidales des contaminants qui ne seront plus mobilisables, sauf actions anthropiques. L'estuaire filtre activement les apports vers la mer ;

- Pour les contaminants à comportement éminemment dissous comme le lindane, l'atrazine, la simazine, subissant une simple dilution progressive avec les eaux de mer en principe exemptes de contamination, les flux à la mer sont invariants. On trouve ici le rôle de filtre passif de l'estuaire. 
- Enfin, un autre rôle moins connu des estuaires macrotidaux : Celui de régulateur des flux. L'augmentation progressive des sections mouillées et la présence du bouchon vaseux augmente sensiblement le temps de séjour des contaminants dissous et particulaires, et peut absorber les à-coups des apports amont, qu'ils soient liés à une crue ou à un accident.

En résumé, on a toujours comparé à juste titre un estuaire à une station d'épuration. C'est vrai, et elle s'apparente plutôt à une station d'épuration biologique! Sauf que l'important stock de matière organique particulaire biodégradable peut conduire en s'oxydant à un sérieux déficit en oxygène dissous en période estivale. Mais ici il n'y a pas de dispositif efficace de ré-aération...

Plus intéressant encore, les sédiments du bouchon vaseux sont ceux qui contiennent les concentrations les plus faibles en matière organique dans le système fleuve - estuaire - mer. Le temps de séjour important des particules piégées dans le bouchon vaseux, la dégradation progressive du matériel stocké, son «vieillissement " dans le temps, confère a ces particules des caractéristiques remarquables d'homogénéité. L'on peut dire sans trop se tromper qu'un échantillon de sédiments prélevés en Gironde dans la zone du bouchon vaseux aura une concentration en carbone organique proche de $1,5 \%$, en Loire de $3 \%$, en Seine de $4,5 \%$,

De même, et par rapport aux contaminants chimiques adsorbés sur le particulaire, les niveaux de concentrations seront d'autant plus bas dans le système, que la matière organique le sera... Appelons MES $_{\mathrm{bv}}$ (bv pour bouchon vaseux) le type de matériel ainsi identifié.

De ce fait, les facteurs qui contrôlent l'emplacement et la taille du bouchon vaseux ont une influence prépondérante sur la qualité du milieu. Ainsi, les forts étiages permettent la remontée du bouchon vaseux dans des zones amont où la quantité d'eau est plus faible, ce qui induit une dégradation supplémentaire de la qualité des eaux... L'écrêtage des crues empêche l'évacuation vers la mer d'une partie du matériel stocké dans le bouchon vaseux. Plus globalement, tout aménagement amont et interne à l'estuaire (extraction de sables et graviers, endiguement, aménagements portuaires) aura aussi des conséquences sur la qualité du milieu estuarien. Le cas des dragages sera traité par la suite.

\section{VI — LA GESTION DES SÉDIMENTS DANS CE TYPE DE MILIEU}

La question du cycle hydro-sédimentaire estuarien, du rôle du bouchon vaseux sur la modification des flux et de l'homogénéité étonnante des concentrations en contaminants dans le particulaire devrait conduire à une appréciation particulière des problèmes induits par l'effet des dragages en estuaire.

Nous pouvons envisager le problème des dragages sous cinq aspects différents... Les effets sur le milieu :

- De la remise en suspension de matériel particulaire du fait de dragages d'approfondissement... Et dans ce cas on mobilisera du sable mélangé à de l'ancien $\mathbf{M E S}_{\mathbf{b v}}$.

- De la remise en suspension de matériel particulaire du fait des dragages d'entretien des chenaux... Et dans ce cas on mobilisera souvent du sable mélangé à du $\mathbf{M E S}_{\mathbf{b v}}$. Le cas du dragage à l'américaine étant le plus critique.

- De la remise en suspension de matériel particulaire du fait des dragages dans les bassins portuaires. Dans ce cas on mobilisera aussi du sable mélangé à de l'ancien $\mathbf{M E S}_{\mathbf{b v}}$, qui par ailleurs a pu être parfois soumis à des rejets directs d'origine industrielle ou portuaire. Ceci peu correspondre au cas précédent.

- Des dépôts des boues de dragage dans des sites internes ou externes au système estuarien... On associe souvent à ce choix le problème du coût du transport de ces matériaux.

- De l'évolution morphologique de l'estuaire. En effet, d'un point de vue strictement géomorphologique, les grandes quantités de matériel sédimentaire mobilisé par les ports sont de nature à modifier les volumes internes estuariens et indirectement le fonctionnement du système bouchon vaseux - crème de vase - vasières. On agira dans ce cas directement sur la valeur du MES $_{\text {bv }}$. Par exemple, accélérer le comblement de l'estuaire veut dire un temps de séjour moindre pour le matériel particulaire, une expulsion plus fréquente en période de crue... En fait, ceci correspond à diminuer le rendement de la station d'épuration biologique (particulièrement le cycle de la matière organique...).

\section{VII — CONSIDÉRATIONS PHILOSOPHIQUES ET CONCLUSION}

Dans ce même séminaire, C. Alzieu traitera des aspects spécifiques à la gestion des dragages dans le milieu marin et estuarien. Je souhaite seulement placer ici les cas 2,3 et 4 dans un contexte plus général et même philosophique.

Nous avons indiqué précédemment qu'un estuaire pouvait se comporter comme une station d'épuration pour la matière organique et les contaminants chimiques. De plus, du fait des fortes turbidités présentes, toute zone de basse énergie induira une sédimentation importante de ce matériel fin: zones intertidales, engainement des chenaux, ports industriels, ports de plaisance... Le taux de sédimentation devient d'autant plus important que le système est confiné. Nous nous trouvons confrontés ici à l'aspect décantation de la station d'épuration. Dans le cas ou cette décantation ne peut avoir lieu, soit parce qu'il n'y pas de décanteurs, soit parce que l'estuaire est en voie de comblement (cas de nombreux estuaires de la Manche), le matériel fin poursuivrait son cheminement vers la mer après avoir séjourné quelque temps dans l'estuaire.

La présence simultanée d'un matériel fin reflétant la contamination moyenne du bassin versant et ses apports $\left(\mathbf{M E S}_{\mathbf{b v}}\right)$, la présence d'un système de décantation (port), le besoin de gérer correctement ce type de matériel (dragages) replace ce problème, toutes proportions gardées, dans le droit fil des problèmes liés à la gestion des boues de station d'épuration !

Laisser poursuivre le cheminement de ce matériel vers le milieu marin (ce qui aurait été le cas sans système de décantation !), et alors le dragage à l'américaine et les rejets en mer (après dispersion) des boues de dragage auraient légitimement leur place...

Ou alors il faudrait ne plus rejeter les boues dans le milieu, et on rejoint l'ensemble des gestionnaires de barrages, chenaux, et ports maritimes dans la recherche de techniques de traitement ou de zones de dépôts adéquates à terre...

Quel choix doit-il être fait ? Cela devient moins un problème scientifique et technique qu'un problème de société...

\section{BIBLIOGRAPHIE}

[1] Prtrchard D.W. (1967), - What is an Estuary: Physical View-point. In : Estuaries. American Association for Advancement of Science, Washington. G.H. Lauff (ed.), vol. 83, 3-5. 
[2] FaIRBridge R.W. (1980). - The estuary: its definition and chemical role. In: Chemistry and Biochemistry of estuaries. E.O.I. Cato (ed.), Jhon Wiley and Sons, Chichester, 1-35.

[3] Thouvenin B., Le Hir P., Maurice L., Romañ L.A. (1997). Synthèse des études relatives à la modélisation de l'oxygène dissous dans l'estuaire de la Loire. Rapport de synthèse de I'APEEL - 1984-1994.

[4] Romasa L.A. (1997). - Le potentiel de recherche français sur les estuaires: comment le mobiliser sur les besoins identifiés lors du séminaire - Les Estuaires Français : évolution naturelle et artificielle des estuaires français. Actes de colloque - 22 : 320-328.
[5] Romaña L.A. (1999). - Rôle de l'estuaire dans le stockage et le flux des contaminants issus de l'amont. Recherches Marines $\mathrm{n}^{\circ}$ 22. Avril 1999.

[6] Romaña L.A., Bediot G. (2000). - L'estuaire de la Seine: fonctionnement, perspectives. Revue Elsevier Vol $8 \mathrm{n}^{\circ} 2$ avriljuin 2000. Natures Sciences Sociétés.

[7] Hamm L.. Romana L.A.. Lerat F. - Colloque Restauration des Ecosystèmes côtiers 08-09 novembre 2000 à Brest « Maintien des fonctionnalités biologiques de la vasière nord de l'estuaire de la Seine ». En cours d'impression (Ed; Ifremer). 